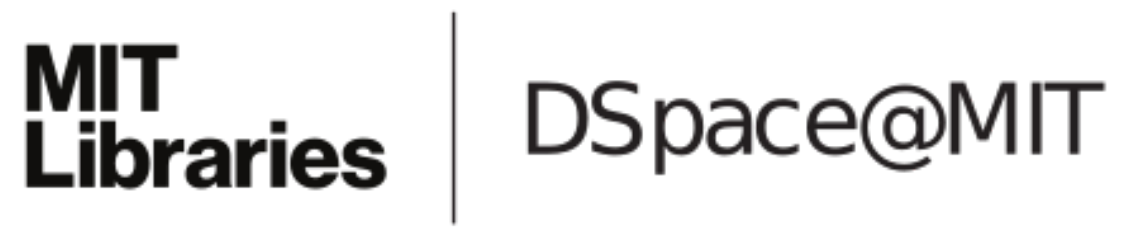

\author{
MIT Open Access Articles
}

\section{Assessment of lower extremity motor adaptation via an extension of the Force Field Adaptation Paradigm}

The MIT Faculty has made this article openly available. Please share how this access benefits you. Your story matters.

Citation: Cajigas, I. et al. "Assessment of lower extremity motor adaptation via an extension of the Force Field Adaptation Paradigm." IEEE, 32nd Annual International Conference of the IEEE EMBS, Buenos Aires, Argentina, August 31 - September 4, 2010. 4522-4525.๑ 2011 IEEE.

As Published: http://dx.doi.org/10.1109/iembs.2010.5626058

Publisher: Institute of Electrical and Electronics Engineers

Persistent URL: http://hdl.handle.net/1721.1/67498

Version: Final published version: final published article, as it appeared in a journal, conference proceedings, or other formally published context

Terms of Use: Article is made available in accordance with the publisher's policy and may be subject to US copyright law. Please refer to the publisher's site for terms of use. 


\title{
Assessment of Lower Extremity Motor Adaptation via an Extension of the Force Field Adaptation Paradigm
}

\author{
Iahn Cajigas, Student Member, IEEE, Mary T. Goldsmith, Student Member, IEEE, Alexander Duschau-Wicke, \\ Student Member, IEEE, Robert Riener, Member, IEEE, Maurice A. Smith, Emery N. Brown, Fellow, IEEE, \\ Paolo Bonato, Senior Member, IEEE
}

\begin{abstract}
Lower extremity rehabilitation has seen recent increased interest. New tools are available to improve gait retraining in both adults and children. However, it remains difficult to determine optimal ways to plan interventions due to difficulties in continuously monitoring outcomes in patients undergoing rehabilitation. In this paper, we introduce an extension of the Force Field Adaptation Paradigm, used to quantitatively assess upper extremity motor adaptation, to the lower extremity. The algorithm is implemented on the Lokomat lower extremity gait orthosis and utilized to assess short-term motor adaptation. Establishing an understanding of how healthy adults' motor systems adapt to external perturbations will be important to understanding how the adaptive mechanisms involved in gait are altered by disease.
\end{abstract}

\section{INTRODUCTION}

$\mathrm{T}$ HE field of lower extremity rehabilitation has recently witnessed a surge in interest. Devices such as the PAM/POGO [1], the LOPES [2], and the LOKOMAT [3] have played a key role in this trend. Robots allow highly repeatable interventions and serve as instruments to quantify the rehabilitation progress. Despite the current high cost of many of these devices, the introduction of FDA-approved robotic gait retraining devices in the clinic is an important step toward assessing the efficacy of these tools via randomized multi-centered clinical trials.

Initial studies of robotic technologies for lower extremity rehabilitation interventions show high variability in individual responses [4-7]. Many aspects of robot-assisted gait training could contribute to the differences seen in motor gains across subjects. The level of patient engagement during the rehabilitation session is a factor associated with the magnitude of the observed motor gains. Several researchers have hypothesized that the patient's baseline

Manuscript received April 25, 2010.This work was supported in part by the National Institute of Neurological Disorders and Stroke under the grant entitled "Automated and Adaptive Lower Extremity Neuro-Rehabilitation for Stroke" (award \# 1F31NS058275-01A2).

I. Cajigas and E.N. Brown are with the Harvard-MIT Department of Health Sciences and Technology (e-mail: $\underline{\text { iahn@mit.edu; }}$ enb@neurostat.mit.edu).

M.T. Goldsmith is with the Department of Biomedical Engineering at Boston University (e-mail: marygold@bu.edu).

A. Duschau-Wicke and R. Riener are with the Sensory-Motor Systems (SMS) Lab, Institute of Robotics and Intelligent Systems (IRIS), ETH Zurich \&Medical Faculty, Balgrist University Hospital, University of Zurich, Switzerland (e-mail: duschau@mavt.ethz.ch, riener@mavt.ethz.ch)

A. Duschau-Wicke is also with Hocoma AG, Volketswil, Switzerland.

I. Cajigas and P. Bonato are with the Department of Physical Medicine and Rehabilitation, Harvard Medical School and Spaulding Rehabilitation Hospital, Boston, MA, 02114, USA (corresponding author; phone: 617573-2770; fax: 617-573-2769; e-mail: pbonato@partners.org). condition (i.e. pre-rehabilitation functional state and impairment level) correlates with the magnitude of observed motor gains. Additionally, mechanical constraints imposed by the robotic system itself (e.g. limitations in the degreesof-freedom imposed at the pelvis) may play a role in the observed variability in rehabilitation outcomes across patients. Since motor adaptation refers to learning through the alteration of motor commands in response to changes in the environment, by quantifying the adaptive process during rehabilitation one can gain insight into the factors that govern this variability. One would anticipate that adaptations observed during performance of the training session in response to forces generated by the robotic system would be predictive of rehabilitation outcomes.

The goal of the work herein presented is to develop a platform to quantify the motor adaptations that occur during gait retraining - one of the above-mentioned factors that we see as responsible for the observed variability in rehabilitation outcomes following robot-assisted gait training. Specifically, we developed a methodology to characterize the rates of lower extremity motor adaptation in a group of healthy adults. The proposed methodology is an extension of a paradigm originally used to test motor adaptation in the upper extremities. The basis for this paradigm is the introduction of velocity-dependent force perturbations perpendicular to the direction of movement that induce trajectory errors to which the subjects must then adapt. Measurement of a subject's adaptive compensatory forces and restoration of movement kinematics during and after the removal of these perturbations can be used to determine the rates of motor adaptation. In future studies, we plan to establish a methodology for comparing motor adaptations between healthy individuals and patients based on the work herein presented. The methodology will also provide a potential tool for assessing the efficacy of different rehabilitation methodologies, in terms of the rates of adaption and the retention of motor skills.

\section{METHODS}

\section{A. Force Field Adaptation Paradigm}

Shadmehr and Mussa-Ivaldi [8] studied how the central nervous system adapts to changing environment dynamics as the hand interacts with a novel mechanical environment, i.e. an applied force field, using the force field adaptation paradigm (FFAP). In their experimental methodology, a practice period allows subjects to practice reaching while holding the end-effector (or end-point) of the robotic arm in 
a force free condition (Baseline/Null-Field Movement). After this practice period, a perturbing velocity-dependent force is applied perpendicular to the direction of motion. That is,

$$
\mathbf{F}(t)=\left[\begin{array}{l}
F_{x} \\
F_{y}
\end{array}\right]=\left[\begin{array}{cc}
0 & -B \\
0 & 0
\end{array}\right] \cdot\left[\begin{array}{l}
v_{x} \\
v_{y}
\end{array}\right]
$$

where $\mathbf{F}(t)$ is the vector of end-effector forces, $F_{x}$ and $F_{y}$ are the $x$ and $y$ components of the force, $B$ is the viscous damping coefficient, and $v_{x}$ and $v_{y}$ are the $x$ and $y$ components of the end-effector velocity. During straight line movements, $v_{x}=0$ and $v_{y}$ is approximately bell-shaped leading to a force in the negative $x$-direction that is also bell-shaped and proportional to $v_{y}$. During force field movements, subjects are instructed to continue to perform straight line movements at their original movement velocity. Over time, subjects adapt to the force field and re-achieved the baseline trajectory by generating compensatory forces. Upon removal of the force field and return to the baseline condition, the compensatory forces that subjects learn to apply drive their arms along trajectories that mirror the path their limbs would have followed had they not compensated for the applied force field. This mirroring, or after-effect, suggests the development of an internal model during the process of adaptation.

The time course of the after-effect can be quantified by the perpendicular displacement from the baseline path at the point of maximum velocity/force. If $\hat{i}$ and $\hat{j}$ are unit vectors in the $x$ and $y$ directions, then we define the perpendicular deviation from the baseline path as

$$
d_{\perp}=\left\langle\hat{i}, \overrightarrow{\mathbf{p}}_{\mathbf{n}}-\overrightarrow{\mathbf{p}}_{\mathbf{0}}\right\rangle=x-x_{0}
$$

where $\left\langle\mathbf{x}_{1}, \mathbf{x}_{2}\right\rangle=\mathbf{x}_{1}{ }^{T} \cdot \mathbf{x}_{2}$ is the standard Cartesian inner product, $\mathbf{x}_{i}{ }^{T}$ denotes the transpose of the vector $\mathbf{x}_{i}, \mathbf{p}_{\mathbf{n}}$ is the position vector of the end-effector at $v_{\max }$ during the $n^{\text {th }}$ trial, and $\mathbf{p}_{\mathbf{0}}$ is the position vector at $v_{\max }$ during the mean of the baseline trials.

Later experiments by Scheidt et al. [9] introduced errorclamp (EC) movements during which the subject's arm is limited to move along the original straight line trajectory. The result of error clamp trials is a minimization of the trajectory error between the original and current movement paths. Error clamp movements, interspersed during the force field condition, offer a window into the time course of the adaptive process by measuring the subject's lateral compensatory forces during imposed straight reaching movements. These measured forces can be compared against the applied force field, to determine how well the subject has adapted to the applied force field during the learning block condition. The coefficient of adaptation, a measure of the subject's current level of adaptation, is defined as

$$
F_{x}^{n}(t)=c_{\text {adapt }} \cdot F_{x}^{\text {ideal }}(t)
$$

where $F_{x}^{n}(t)$ is the force measured during the $n^{\text {th }}$ error clamp trial, $F_{x}^{\text {ideal }}(t)$ is the ideal compensatory lateral force computed from (1) using the average velocity in the $y$ -

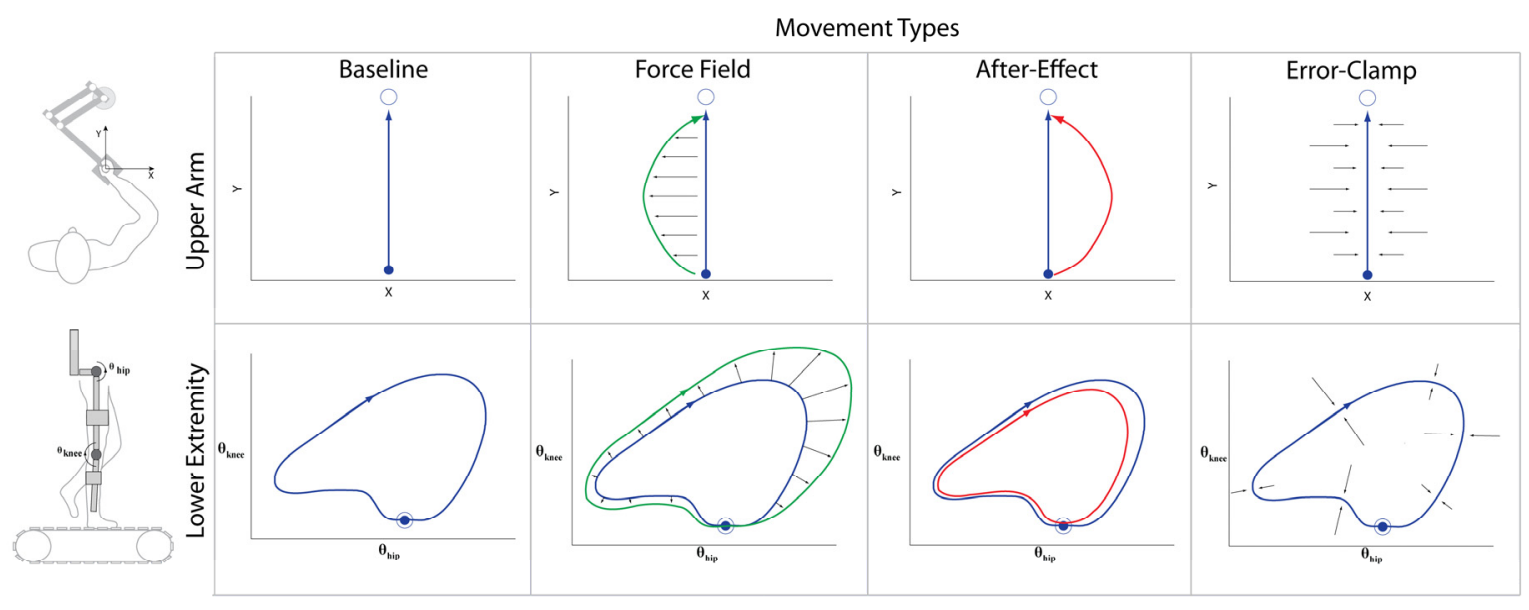

Figure 1. Comparison between the lower and upper extremity FFAP. (leftmost) Baseline Movements: In the upper extremity FFAP, subjects are asked to make rapid point-to-point movements to a target. In the lower extremity, subjects are asked to walk normally at a specified treadmill speed. The gait cycle can be considered a point-to-point movement where the beginning and end points coincide. (left of center) Force Field: In the upper extremity FFAP, a velocity dependent force field is applied perpendicular to the direction of movement. In the lower extremity FFAP, unit vectors along the baseline path can be used to define instantaneous coordinate systems oriented parallel and perpendicular to the path. Velocity-dependent force/torque perturbations are then applied along the instantaneous perpendicular direction. (right of center)After-Effect: In the upper and lower extremity FFAP removal of the force field results in a movement that is the mirror image of the path their limbs would have followed had they not compensated for the applied force field. (right) Error Clamp Movements: Upper Extremity FFAP/Lower Extremity FFAP - measurement of the interaction forces while the subject is forced to move along their original baseline path allows the adaptive forces to be assessed over time. These compensatory forces/torques can be compared to the ideal compensatory force (defined by the baseline velocity of the subject during baseline movements). Error clamps can be randomly interspersed within the baseline, force field, and unlearning phases at an occurrence of $20 \%$ in order to measure forces prior, during, and after adaptation. 
direction during baseline trials, and $c_{\text {adapt }}$, is the coefficient of adaptation.

\section{B. Lower Extremity Force Field Adaptation Paradigm}

Extension of the FFAP is a natural approach for testing motor adaptation in the lower limb. Yet the implementation of this paradigm carries a number of intrinsic difficulties for a lower limb system. Our extension of the FFAP to the lower extremity (LE-FFAP) relies on the following observations:

1. The cyclical gait pattern can be considered a point-topoint movement where the start and end points coincide. With this observation, in any coordinate system (joint, end-effector/ankle, Cartesian coordinates, etc.), the standard gait pattern is the curvilinear equivalent to the upper extremity straight line movements.

2. In the lower extremity, the subject's baseline gait pattern defines which movements are parallel to the baseline path. At each point along the gait pattern, a perpendicular direction to the path can be determined and perturbations can be applied in this direction. We define $\mathbf{u}_{\|}(k)=\left[\begin{array}{ll}u_{\| \theta_{h}}(k) & u_{\| \theta_{k}}(k)\end{array}\right]^{T}$ and $\mathbf{u}_{\perp}(k)=\left[\begin{array}{ll}u_{\perp \theta_{h}}(k) & u_{\perp \theta_{k}}(k)\end{array}\right]^{T}$ as the parallel and perpendicular unit vectors at the $k^{\text {th }}$ point along the gait cycle. Then

$$
\mathbf{T}_{k}=\left[\begin{array}{cc}
u_{\| \theta_{h}}(k) & u_{\| \theta_{k}}(k) \\
u_{\perp \theta_{h}}(k) & u_{\perp \theta_{k}}(k)
\end{array}\right]
$$

is the transformation matrix from hip and knee coordinates to "path" coordinates at the $k^{\text {th }}$ point along the gait cycle. In other words, $\mathbf{T}_{k}$ projects the instantaneous velocity vector in joint coordinates at $k^{\text {th }}$ point of the current gait cycle into the corresponding parallel and perpendicular components with respect to the baseline path.

The velocity dependent perturbation torque is then

$$
\begin{gathered}
\boldsymbol{\tau}(k)=\left[\begin{array}{c}
\tau_{h}(k) \\
\tau_{k}(k)
\end{array}\right]=\mathbf{T}_{k}{ }^{\mathbf{T}} \cdot\left[\begin{array}{ll}
0 & 0 \\
B & 0
\end{array}\right] \cdot \mathbf{T}_{k}\left[\begin{array}{l}
v_{h} \\
v_{k}
\end{array}\right] \\
\boldsymbol{\tau}(k)=\mathbf{T}_{k}^{\mathbf{T}} \cdot \mathbf{B} \cdot \mathbf{T}_{k} \cdot \mathbf{v}
\end{gathered}
$$

where $\tau_{h}$ and $\tau_{k}$ are the hip and knee components of the joint torque vector $\tau(k), B$ is the viscous damping coefficient, and $v_{h}$ and $v_{k}$ are the hip and knee components of the joint velocity vector, $\mathbf{v}$.

3. Compensation to the applied perturbations can be assessed by forcing the subject to walk along their baseline gait trajectory and measuring the generalized interaction forces perpendicular to the baseline path. This is accomplished through implementation of errorclamp trials.

During error-clamp movements, the joint torques $\boldsymbol{\tau}(k)$ required to maintain the subject moving along their baseline path is equal and opposite to the torques applied by the subject. Because the perturbing force field is oriented perpendicular to the baseline path, the perpendicular component of the interaction torque during the $n^{\text {th }}$ trial, $\tau_{\perp}{ }^{n}$, represents the current adaptive compensatory force. This component, $\tau_{\perp}{ }^{n}$, is obtained by projecting the joint torques onto the instantaneous perpendicular direction along the baseline path, i.e.

$$
\tau_{\perp}^{n}(k)=\left\langle\mathbf{u}_{\perp}, \boldsymbol{\tau}(k)\right\rangle
$$

The adaptation coefficient is then defined as in (3)

$$
\tau_{\perp}^{n}(k)=c_{\text {adapt }} \cdot \tau_{\perp}^{\text {ideal }}(k)
$$

where $\tau_{\perp}^{\text {ideal }}(k)=B \cdot v_{\|}^{B L}(k)$ is determined from the viscous coefficient $\mathrm{B}$ and the parallel velocity component during mean baseline locomotion.

The time course of the after-effect can be quantified by the perpendicular displacement from the baseline path at the point of maximum velocity/force. We define the perpendicular deviations from the baseline path as

$$
d_{\perp}=\left\langle u_{\perp}, \overrightarrow{\mathbf{p}}_{\mathbf{n}}-\overrightarrow{\mathbf{p}}_{\mathbf{0}}\right\rangle
$$

where $\mathbf{p}_{\mathbf{n}}$ is the position vector at $v_{\max }$ during the $n^{\text {th }}$ trial, and $\mathbf{p}_{\mathbf{0}}$ is the position vector at $v_{\max }$ during the mean baseline trials. Fig. 1 shows a comparison between lower and upper extremity FFAP.

\section{Implementation}

The FFAP of Shadmehr and Mussa-Ivaldi [8] was extended to the lower extremity and implemented on the Lokomat lower extremity gait orthosis (Hocoma AG, Switzerland). The LE-FFAP begins with the collection of pre-baseline data with the Lokomat in a highly back-drivable state, which allows the subject to move freely within the Lokomat. Implementation of a new Lokomat mode of operation, termed Path Control [10], allows subjects to naturally control the timing of their gait. The path control algorithm relies on the synchronization of the current gait cycle with a normalized reference gait cycle. This synchronization is accomplished via the algorithm of Aoyagi et al. [11]. The baseline condition of the LE-FFAP requires subjects to walk as freely as possible while attached to the Lokomat. Device loading of the subject was minimized via the generalized elasticity method of Vallery et al. [12]. This procedure utilizes the subject's own pre-baseline gait cycle and a model of the Lokomat dynamics to generate an elastic force field that minimizes subject effort required to move the orthosis along the gait trajectory while maintaining passivity of the control system.

\section{Experimental Procedures}

During the pre-baseline period, joint kinematics are recorded via potentiometers on the Lokomat. From prebaseline data, an average trajectory in the joint space is obtained. This information is used to generate a set of 
generalized elasticity coefficients that compensate for the machine dynamics via the method of Vallery et al. [12]. Additionally unit vectors parallel and perpendicular to the path are computed and used to determine the perturbation torques to be applied according to (5).

The experimental procedure is broken into 3 blocks:

1) Baseline: the subject moves freely in a force-free condition

2) Force Field: the subject is able to walk freely, but with the addition of a velocity-dependent force field that is applied perpendicular to the path of motion.

3) Unlearning : Return to a force-free condition.

The Lokomat is set to a selected speed of $3.0 \mathrm{~km} / \mathrm{hr}$ for the duration of the experiment. The viscous damping coefficient, $B$, was set to $3.5 \mathrm{~N} \cdot \mathrm{sec} / \mathrm{rad}$. For brevity, error-clamp results are not presented herein.

\section{RESULTS}

Fig. 2 shows the results for a single subject. Panel A shows the joint trajectories during Baseline, Force Field, Unlearning phases of the experiment. There were 100 steps in each phase. Panel B shows the mean baseline curve from Panel A, the mean of the first 10 steps in Force Field, and the mean of the first 10 steps in the Unlearning phase of the experiment. Panel $\mathrm{C}$ shows the perpendicular deviation from the mean baseline path at the point where the parallel velocity to the path is maximum, $d_{\perp}$. The dark curves are used to illustrate the trends in the data.

$$
d_{\perp}=\left\{\begin{array}{lc}
0 & 1<n<100 \\
2.6 \times 10^{-2} \cdot e^{\frac{-t}{84}} & 101<\mathrm{n}<200 \\
3.0 \times 10^{-2} \cdot e^{\frac{-t}{111}} & 201<\mathrm{n}<300
\end{array}\right.
$$

This piecewise regression on $d_{\perp}$ yields an $R^{2}$ of $77 \%$. Observation of the time constants for the Force Field and Unlearning phases of the experiment suggests that the
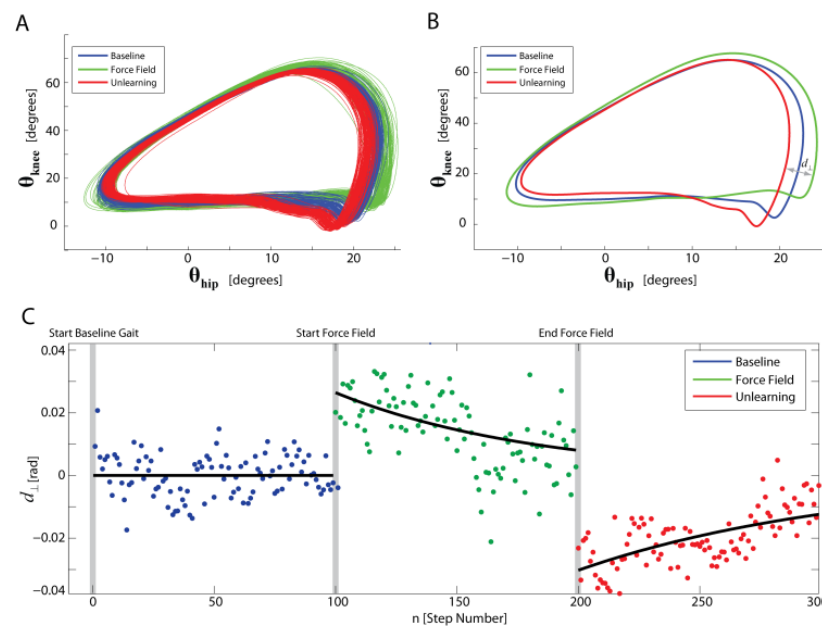

Figure 2 A) Joint trajectories during Baseline, Force Field, and Unlearning phases of experiment. B) Mean baseline trajectory vs. early force field and early after-effects locomotion. C) Perpendicular deviation from the baseline path at the point of maximum velocity (indicated in B). unlearning process occurs at a slightly slower rate than the adaptation process in this subject.

\section{CONCLUSIONS}

The work herein presented demonstrates how the LEFFAP can be used to assess lower extremity motor adaptation. Preliminary results validate the methodology by demonstrating after-effects analogous to those seen in the upper extremity. Future work will include analysis of both after-effects and the coefficient of adaptation in a group of healthy adult volunteers. Establishing an understanding of how healthy adults' motor systems adapt to external perturbations will be an important step towards understanding how the adaptive mechanisms involved in gait are altered by disease.

\section{ACKNOWLEDGEMENTS}

The authors would like to thank Lars Lünenburger, Heike Vallery, L. Nicolas Gonzalez Castro, and Gary Sing for their contributions.

\section{REFERENCES}

[1] D. J. Reinkensmeyer, et al., "Tools for understanding and optimizing robotic gait training," J Rehabil Res Dev, vol. 43, pp. 657-70, AugSep 2006.

[2] J. F. Veneman, et al., "Design and evaluation of the LOPES exoskeleton robot for interactive gait rehabilitation," IEEE Trans Neural Syst Rehabil Eng, vol. 15, pp. 379-86, Sep 2007.

[3] G. Colombo, et al., "Treadmill training of paraplegic patients using a robotic orthosis," J Rehabil Res Dev, vol. 37, pp. 693-700, Nov-Dec 2000.

[4] J. Hidler, et al., "Multicenter randomized clinical trial evaluating the effectiveness of the Lokomat in subacute stroke," Neurorehabil Neural Repair, vol. 23, pp. 5-13, Jan 2009.

[5] T. G. Hornby, et al., "Enhanced gait-related improvements after therapist- versus robotic-assisted locomotor training in subjects with chronic stroke: a randomized controlled study," Stroke, vol. 39, pp. 1786-92, Jun 2008

[6] B. Husemann, et al., "Effects of locomotion training with assistance of a robot-driven gait orthosis in hemiparetic patients after stroke: a randomized controlled pilot study," Stroke, vol. 38, pp. 349-54, Feb 2007.

[7] A. Mayr, et al., "Prospective, Blinded, Randomized Crossover Study of Gait Rehabilitation in Stroke Patients Using the Lokomat Gait Orthosis," Neurorehabil Neural Repair, vol. 21, pp. 307-314, July 1, 20072007.

[8] R. Shadmehr and F. A. Mussa-Ivaldi, "Adaptive representation of dynamics during learning of a motor task," J Neurosci, vol. 14, pp. 3208-24, May 1994.

[9] R. A. Scheidt, et al., "Persistence of motor adaptation during constrained, multi-joint, arm movements," J Neurophysiol, vol. 84, pp. 853-62, Aug 2000.

[10] A. Duschau-Wicke, et al., "Path Control: A Method for PatientCooperative Robot-Aided Gait Rehabilitation," Neural Systems and Rehabilitation Engineering, IEEE Transactions on, vol. 18, pp. 38-48, 2010.

[11] D. Aoyagi, et al., "A robot and control algorithm that can synchronously assist in naturalistic motion during body-weightsupported gait training following neurologic injury," IEEE Trans Neural Syst Rehabil Eng, vol. 15, pp. 387-400, Sep 2007.

[12] H. Vallery, et al., "Generalized elasticities improve patientcooperative control of rehabilitation robots," in Rehabilitation Robotics, 2009. ICORR 2009. IEEE International Conference on, 2009, pp. 535-541. 